\title{
Stokes Representation for the Solutions of Maxwell-Vlasov
}

\author{
Monica Pîrvan, Constantin Udrişte
}

University Politehnica of Bucharest, Department of Mathematics I, Splaiul Independeței, Bucharest, Romania.

Email: \{anet.udri, monicapirvan\}@yahoo.com

Received September $25^{\text {th }}, 2010$; revised November $12^{\text {th }}, 2010$; accepted December $15^{\text {th }}, 2010$.

\begin{abstract}
Maxwell-Vlasov PDEs system describes the dynamics of plasma consisting of charged particles with long-range interaction. Their solutions can be written using some Stokes potentials. Section 1 presents the experimental devices which can produce a magnetic trap. Magnetic geometric dynamic provides mathematical tools for describing the magnetic flow (see [1-7]). Stokes representation for the solutions of PDEs as Maxwell PDEs or Maxwell-Vlasov PDEs are used analyzing electromagnetic energy in magnetic traps. Section 2 studies Maxwell-Vlasov PDEs system. Stokes representation of its solutions, using Maximum Principle for a multitime optimal control problem, is obtained. Section 3 discusses a method for changing a given ODEs system into a geodesic motion under a gyroscopic field of forces (geometric dynamics). Section 4 proposes a modified form for Maxwell-Vlasov PDEs, by replacing the classical gyroscopic force with the one appearing in geometric dynamics. Stokes representation for the solutions of modified Maxwell-Vlasov PDEs is also obtained.
\end{abstract}

Keywords: Maxwell-Vlasov PDEs, Magnetic Traps, Dynamics of Plasma, Gyroscopic Field of Forces, Dynamic Systems

\section{Magnetic Traps}

Our theory is appropriate for the study of electromagnetic energy in magnetic traps. These devices are used for atoms or charged particles:

1) magnetic traps, used to trap neutral atoms in a magnetic field gradient:

2) Penning trap, used to trap charged particles or ions in a combination of electrostatic potential and uniform magnetic field;

3) magneto-optical trap (or MOT), a trap using a magnetic gradient and laser to trap neutral atoms;

4) magnetic tweezers, a trap using a magnetic field to trap micrometreseized ferromagnetic beads.

The first magnetic trap was realized by $\mathrm{M}$. Ioffe for plasma confination having in mind the following idea: if an atom is not too energetic, it can be held in a magnetic nonzero minimum - a region from which the strength of the magnetic field grows stronger in every direction [3]. Other modern magnetic traps, analyzed from the perspective of geometric dynamics in [6,7], are used today in EDM experiments or for creating an ECR source of MCI.

The ALPHA experiment at CERN [8] seeks to trap antihydrogen atoms inside a magnetic bottle consisting of a super-conducting octupole magnet and two mirror coils. We do it with superconducting magnets - mirror coils to create a minimum in the middle of the trap's axis, plus an octupole magnet to create a minimum in the center of the trap's radius. To study the energy spectra of antihydrogen atoms it will be necessary to keep them from blowing themselves up for much longer than a few thousandths of a second currently possible - then will have to be detained for at least a few seconds. Luckily, even neutral atoms have a small magnetic moment; they can be confined by a magnetic field of the right shape and strength. The current ALPHA plan uses magnetic fields specially shaped by an octupole magnet as part of the trap. The design of ALPHA magnetic trap was developed and refined at Berkeley Lab by a large team of Berkeley Lab and UC Berkeley scientists, visitors and students.

\section{Stokes Representations for the Solutions of Maxwell-Vlasov PDEs System}

The ideas of this paper start from the papers [1-20] and from the conversations with D. Wang about the VlasovMaxwell-Boltzmann PDEs, carried at Siam Conference on Analysis of Partial Differential Equations, December 
7-10, 2009, Miami, Florida.

We consider the case of collisionless plasma, i.e., the case of a single species of particles with mass $m$ and charge $e$.

Let

$$
\Omega_{\left(t_{0}, x_{0}, v_{0}\right),\left(t_{f}, x_{f}, v_{f}\right)}=\Omega_{\left(t_{0}, x_{0}\right),\left(t_{f}, x_{f}\right)}^{1} \times \Omega_{\left(v_{0}, v_{f}\right)}^{2}
$$

be a hyperparallelipiped in $R^{7}$ determined by the opposite diagonal points $\left(t_{0}, x_{0}, v_{0}\right)$ and $\left(t_{f}, x_{f}, v_{f}\right)$.

If $E(t, x)$ represents a $\mathbf{C}^{1}$ electric field, $B(t, x)$ represents a $\mathbf{C}^{1}$ magnetic field and $L(t, x, v)$ is $\mathbf{C}^{1}$ plasma particle number density per phase space $(v=\dot{x}$ being the velocity), Maxwell-Vlasov PDEs system is

$$
\begin{aligned}
& \frac{\partial L}{\partial t}+\left\langle v, \frac{\partial L}{\partial x}\right\rangle+\frac{e}{m}\left\langle E+\frac{1}{c} v \times B, \frac{\partial L}{\partial v}\right\rangle=0, \\
& \frac{1}{c} \frac{\partial B}{\partial t}=-\operatorname{curl} E, \frac{1}{c} \frac{\partial E}{\partial t}=\operatorname{curl} B-\frac{1}{c} j, \\
& \operatorname{div} E=\rho, \operatorname{div} B=0,
\end{aligned}
$$

where $c$ is the speed of light, $j$ is the current defined by $L$,

$$
j(t, x)=e \int_{\Omega_{\left(v_{0}, v_{f}\right)}^{2}} L(t, x, v) v d v
$$

and $\rho$ is the charge density,

$$
\rho(t, x)=e \int_{\Omega_{\left(v_{0}, v_{f}\right)}^{2}} L(t, x, v) d v
$$

Theorem 1. Let $\Omega_{\left(t_{0}, x_{0}, v_{0}\right),\left(t_{f}, x_{f}, v_{f}\right)}$ be the parallelepiped fixed by two diagonal points $\left(t_{0}, x_{0}, v_{0}\right)$ and $\left(t_{f}, x_{f}, v_{f}\right)$ and

$(t, x, v)=\left(\tilde{N}(t, x, v), \bar{N}(t, x, v), n^{7}(t, x, v)\right)$ be the unit normal vector of the boundary $\partial \Omega_{\left(t_{0}, x_{0}, v_{0}\right),\left(t_{f}, x_{f}, v_{f}\right)}$. The solutions of Maxwell - Vlasov PDEs (1) admit the Stokes representation

$$
\begin{aligned}
E(t, x)= & \operatorname{curl} p(t, x)-\frac{1}{c} \frac{\partial q}{\partial t}-\operatorname{grad} \alpha(t, x) \\
& -\frac{e}{m} L(t, x, v) \operatorname{grad}_{v} \gamma(t, x, v) \\
B(t, x)= & \operatorname{curl} q(t, x)-\frac{1}{c} \frac{\partial p}{\partial t}-\operatorname{grad} \beta(t, x) \\
& -\frac{e}{m c} L(t, x, v)\left(\operatorname{grad}_{v} \gamma(t, x, v) \times v\right),
\end{aligned}
$$

together with the condition

$$
\begin{aligned}
& \frac{\partial \gamma}{\partial t}+\left\langle\operatorname{grad}_{x} \gamma, v\right\rangle \\
& -\frac{e}{m}\left\langle E+\frac{1}{c} v \times B, \operatorname{grad}_{v} \gamma(t, x, v)\right\rangle=0
\end{aligned}
$$

and the boundary conditions

$$
\begin{aligned}
& \alpha(t, x) \tilde{N}(t, x, v)+p(t, x) \times \tilde{N}(t, x, v) \\
& +\frac{e}{m} \gamma(t, x, v) L(t, x, v) \bar{N}(t, x, v) \\
& +\frac{1}{c} q(t, x) n_{7}(t, x, v) \mid \partial \Omega\left(t_{0}, x_{0}, v_{0}\right),\left(t_{f}, x_{f}, v_{f}\right)=0, \\
& \beta(t, x) \tilde{N}(t, x, v)+q(t, x) \times \tilde{N}(t, x, v) \\
& +\frac{e}{m} \gamma(t, x, v) L(t, x, v) \bar{N}(t, x, v) \\
& +\frac{1}{m c} \gamma(t, x, v) L(t, x, v)(v \times \bar{N}) \\
& +\frac{1}{c} p(t, x) n_{7}(t, x, v) \mid \partial \Omega\left(t_{0}, x_{0}, v_{0}\right),\left(t_{f}, x_{f}, v_{f}\right)=0, \\
& \frac{e}{m} \gamma(t, x, v)\left\langle E+\frac{1}{c} v \times B, \bar{N}\right\rangle \\
& +c \gamma(t, x, v) n_{7}(t, x, v) \mid \partial \Omega\left(t_{0}, x_{0}, v_{0}\right),\left(t_{f}, x_{f}, v_{f}\right)=0,
\end{aligned}
$$

where $p(t, x), q(t, x), \alpha(t, x), \beta(t, x), \gamma(t, x, v)$ are Stokes potentials.

Proof. We consider the following multitime optimal control problem

$$
\begin{aligned}
& \max _{E(\cdot, \cdot)} I(E(\cdot, \cdot))=-\frac{1}{2} \int_{\Omega_{\left(t_{0}, x_{0}, v_{0}\right) \cdot\left(t_{f}, x_{f}, v_{f}\right)}} \\
& \left(\|E(t, x)\|^{2}+\|B(t, x)\|^{2}+\|v\|^{2}\right) d x d v d t,
\end{aligned}
$$

constrained by Maxwell-Vlasov PDE system (1), where the $\mathbf{C}^{1}$ electric field $E(t, x)$ is a control vector function and the $\mathbf{C}^{1}$ magnetic field $B(t, x)$ and the velocity $v$ are state vector functions,

$$
B\left(t_{0}, x_{0}\right)=B_{0}, \quad B\left(t_{f}, x_{f}\right)=B_{f}
$$

Let $p(t, x)=\left(p_{i}(t, x)\right)_{i=\overline{13}}, q(t, x)=(q(t, x))_{i=\overline{1,3}}$, $\alpha(t, x), \beta(t, x), \gamma(t, x, v)$ be $\mathrm{C}^{1}$ functions, considered as co-state variables (Lagrange multipliers) and the Lagrange function

$$
\begin{aligned}
\mathrm{L}^{1} & (t, x, v, E, B, p, q, \alpha, \beta, \gamma) \\
= & -\frac{1}{2}\left(\|E(t, x)\|^{2}+\|B(t, x)\|^{2}+\|v\|^{2}\right) \\
& +\left\langle p(t, x), \frac{1}{c} \frac{\partial B}{\partial t}+\operatorname{curl} E(t, x)\right\rangle \\
& +\left\langle q(t, x), \frac{1}{c} \frac{\partial E}{\partial t}-\operatorname{curl} B(t, x)+\frac{1}{c} j(t, x)\right\rangle \\
& +\alpha(t, x)(\operatorname{div} E(t, x)-\rho(t, x))+\beta(t, x) \operatorname{div} B(t, x) \\
& +\gamma(t, x, v)\left(\frac{\partial L}{\partial t}+v_{1} \frac{\partial L}{\partial x_{1}}+v_{2} \frac{\partial L}{\partial x_{2}}+v_{3} \frac{\partial L}{\partial x_{3}}\right. \\
& \left.+\frac{e}{m}\left\langle E+\frac{1}{c} B \times v, \frac{\partial L}{\partial v}\right\rangle\right) .
\end{aligned}
$$


The optimal control problem (5), with constraints (1), becomes

$$
\begin{aligned}
& \max _{E(\cdot, \cdot)} I(E(\cdot, \cdot))=\int_{\Omega_{\left(t_{0}, x_{0}, v_{0}\right),\left(t_{f}, x_{f}, v_{f}\right)}} \\
& \mathrm{L}(t, x, v, E, B, p, q, \alpha, \beta, \gamma) d x d v d t, \\
& B\left(t_{0}, x_{0}\right)=B_{0}, \quad B\left(t_{f}, x_{f}\right)=B_{f} .
\end{aligned}
$$

Supposing that optimal problem (5), with constraints (1) admits an interior optimal solution $E(t, x)$, we consider the variation $E(t, x, \theta)=E(t, x)+\theta h(t, x)$, where $\theta>0$ and $h(t, x)$ is a $\mathrm{C}^{1}$ arbitrary vector function. We define $B(t, x, \theta)$ and $L(t, x, v, \theta)$ the correspondent state vector function, respectively the plasma particle number density for the variation $E(t, x, \theta)$. For $|\theta|<\theta_{h}$, we construct the Lagrangian $l_{1}(t, x, v, \theta)$ by the formula

$$
\begin{aligned}
& \mathrm{L}(E(t, x, \theta), B(t, x, \theta), L(t, x, v, \theta), p(t, x), \\
&q(t, x), \alpha(t, x), \beta(t, x), \gamma(t, x, v)) \\
&=-\frac{1}{2}\left(\|E(t, x, \theta)\|^{2}+\|B(t, x, \theta)\|^{2}+\|v\|^{2}\right) \\
&+\left\langle p(t, x), \frac{1}{c} \frac{\partial B}{\partial t}(t, x, \theta)+\operatorname{curl} E(t, x, \theta)\right\rangle \\
&+\left\langle q(t, x), \frac{1}{c} \frac{\partial E}{\partial t}(t, x, \theta)-\operatorname{curl} B(t, x, \theta)+\frac{1}{c} j(t, x)\right\rangle \\
&+\alpha(t, x)(\operatorname{div} E(t, x, \theta)-\rho(t, x))+\beta(t, x) \operatorname{div} B(t, x, \theta) \\
&+\gamma(t, x, v)\left(\frac{\partial L}{\partial t}(t, x, v, \theta)+v_{1} \frac{\partial L}{\partial x_{1}}(t, x, v, \theta)\right. \\
&+v_{2} \frac{\partial L}{\partial x_{2}}(t, x, v, \theta)+v_{3} \frac{\partial L}{\partial x_{3}}(t, x, v, \theta) \\
&\left.+\frac{e}{m}\left\langle E(t, x, \theta)+\frac{1}{c} B(t, x, \theta) \times v \frac{\partial L}{\partial v}(t, x, v, \theta)\right\rangle\right) .
\end{aligned}
$$

and the integral function

$$
I(\theta)=\int_{\Omega_{\left(t_{0}, x_{0}, v_{0}\right),\left(t_{f}, x_{f}, v_{f}\right)}} l_{1}(t, x, v, \theta) d x d v d t
$$

It is necessary for $I(\theta)$ to satisfy the condition $I(\theta) \leq I(0),(\forall)|\theta|<\theta_{h}$. If $n(t, x, v)=\left(n^{i}(t, x, v)\right)_{i=\overline{1,7}}$ $=\left(\tilde{N}, \bar{N}, n^{7}\right)$, then

$$
\begin{aligned}
I^{\prime}(\theta) & =\int_{\Omega_{\left(t_{0}, x_{0}, v_{0}\right),\left(t_{f}, x_{f}, v_{f}\right)}}\left[\left(-E_{1}-\frac{\partial p_{2}}{\partial x_{3}}+\frac{\partial p_{3}}{\partial x_{2}}-\frac{1}{c} \frac{\partial q_{1}}{\partial t}-\frac{\partial \alpha}{\partial x_{1}}\right.\right. \\
& \left.-\frac{\partial \gamma}{\partial v_{1}} \frac{e}{m} L\right) h_{1}+\left(-E_{2}-\frac{\partial p_{1}}{\partial x_{3}}-\frac{\partial p_{3}}{\partial x_{1}}-\frac{1}{c} \frac{\partial q_{2}}{\partial t}-\frac{\partial \alpha}{\partial x_{2}}\right. \\
& \left.-\frac{e}{m} \frac{\partial \gamma}{\partial v_{2}} L\right) h_{2}+\left(-E_{3}-\frac{\partial p_{1}}{\partial x_{2}}-\frac{\partial p_{2}}{\partial x_{1}}-\frac{\partial q_{3}}{\partial t} \frac{1}{c}-\frac{\partial \alpha}{\partial x_{3}}\right.
\end{aligned}
$$

$$
\begin{aligned}
& \left.\left.-\frac{e}{m} \frac{\partial \gamma}{\partial v_{3}} L\right) h_{3}\right] d x d v d t \\
& +\int_{\Omega_{\left(t_{0}, x_{0}, v_{0}\right),\left(t_{f}, x_{f}, v_{f}\right)}}\left[\left(-B_{1}-\frac{1}{c} \frac{\partial p_{1}}{\partial t}+\frac{\partial q_{2}}{\partial x_{3}}-\frac{\partial q_{3}}{\partial x_{1}}-\frac{\partial \beta}{\partial x_{1}}\right.\right. \\
& \left.-\frac{e}{m c} L \frac{\partial \gamma}{\partial v_{2}} v_{3}-\frac{e}{m c} L \frac{\partial \gamma}{\partial v_{3}} v_{2}\right) \frac{\partial B_{1}}{\partial \theta}+\left(-B_{2}-\frac{1}{c} \frac{\partial p_{2}}{\partial t}\right. \\
& \left.+\frac{\partial q_{3}}{\partial x_{1}}-\frac{\partial q_{1}}{\partial x_{3}}-\frac{\partial \beta}{\partial x_{2}}-\frac{e}{m c} L \frac{\partial \gamma}{\partial v_{3}} v_{1}+\frac{e}{m c} L \frac{\partial \gamma}{\partial v_{1}} v_{3}\right) \frac{\partial B_{2}}{\partial \theta} \\
& +\left(-B_{3}-\frac{1}{c} \frac{\partial p_{3}}{\partial t}+\frac{\partial q_{1}}{\partial x_{2}}-\frac{\partial q_{2}}{\partial x_{1}}-\frac{\partial \beta}{\partial x_{3}}-\frac{e}{m c} L \frac{\partial \gamma}{\partial v_{1}} v_{2}\right. \\
& \left.\left.+\frac{e}{m c} L \frac{\partial \gamma}{\partial v_{2}} v_{1}\right) \frac{\partial B_{3}}{\partial \theta}\right] d x d v d t \\
& +\int_{\Omega_{\left(t_{0}, x_{0}, v_{0}\right),\left(t_{f}, x_{f}, v_{f}\right)}}\left[-\frac{\partial \gamma}{\partial t}-\frac{\partial \gamma}{\partial x_{1}} v_{1}-\frac{\partial \gamma}{\partial x_{2}} v_{2}-\frac{\partial \gamma}{\partial x_{3}} v_{3}\right. \\
& -\frac{e}{m} \frac{\partial \gamma}{\partial v_{1}} v_{2}\left(E_{1}+\frac{1}{c}\left(B_{3} v_{2}-B_{2} v_{3}\right)\right) \\
& -\frac{e}{m} \frac{\partial \gamma}{\partial v_{2}}\left(E_{2}+\frac{1}{c}\left(B_{1} v_{3}-B_{3} v_{1}\right)\right) \\
& \left.-\frac{e}{m} \frac{\partial \gamma}{\partial v_{3}}\left(E_{3}+\frac{1}{c}\left(B_{2} v_{1}-B_{1} v_{3}\right)\right)\right] \frac{\partial L}{\partial \theta} d x d v d t \\
& +\int_{\partial \Omega_{\left(t_{0}, x_{0}, v_{0}\right),\left(t_{f}, x_{f}, v_{f}\right)}}\left[\left(\alpha n^{1}-p_{3} n^{2}+p_{2} n^{3}+\frac{e}{m} \gamma L n^{4}\right.\right. \\
& \left.+\frac{1}{c} q_{1} n^{7}\right) h_{1} \\
& +\left(p_{3} n^{1}+\alpha n^{2}-p_{1} n^{3}+\frac{e}{m} \gamma L n^{5}+\frac{1}{c} q_{2} n^{7}\right) h_{2} \\
& +\left(-p_{2} n^{1}+p_{1} n^{2}+\alpha n^{3}+\frac{e}{m} \gamma L n^{6}+\frac{1}{c} q_{3} n^{7}\right) h \sigma \\
& +\int_{\partial \Omega_{\left(t_{0}, x_{0}, v_{0}\right),\left(t_{f}, x_{f}, v_{f}\right)}}\left[\left(\beta n^{1}+q_{3} n^{2}-q_{2} n^{3}+\frac{e}{m c} \gamma L v_{3} n^{5}\right.\right. \\
& \left.-\frac{e}{m c} \gamma L v_{2} n^{6}+\frac{1}{c} p_{1} n^{7}\right) \frac{\partial B_{1}}{\partial \theta}+\left(-q_{3} n^{1}+\beta n^{2}+q_{1} n^{3}\right. \\
& \left.-\frac{e}{m c} \gamma L v_{3} n^{4}+\frac{e}{m c} \gamma L v_{1} n^{6}+\frac{1}{c} p_{2} n^{7}\right) \frac{\partial B_{2}}{\partial \theta} \\
& +\left(q_{1} n^{1}+p_{1} n^{2}+\beta n^{3}+\frac{e}{m c} \gamma L v_{2} n^{4}-\frac{e}{m c} \gamma L v_{1} n^{5}\right. \\
& \left.+\frac{1}{c} p_{3} n^{7}\right) \frac{\partial B_{3}}{\partial \theta}+\left(\frac{e}{m} \gamma\left(E_{1}+\frac{1}{c}\left(B_{3} v_{2}-v_{3} B_{2}\right)\right)\right) n^{4} \\
& +\frac{e}{m} \gamma\left(E_{2}\left(B_{1} v_{3}-v_{1} B_{3}\right)\right) n^{5}
\end{aligned}
$$




$$
\left.+\left(\frac{e}{m} \gamma\left(E_{3}+\frac{1}{c}\left(B_{2} v_{1}-v_{2} B_{1}\right)\right) n^{6}+c \gamma n^{7}\right) \frac{\partial L}{\partial \theta}\right] d \sigma .
$$

To impose the condition $I^{\prime}(0)=0$, for any vector function $h(t, x)$, we need a definition for the Lagrangian multipliers via certain PDEs ([9-10]). We obtain the Stokes representation (2), the condition (3) and the boundary conditions (4).

\section{Geodesic Motion in a Gyroscopic Field of Forces}

Let $(M, g)$ be a Riemannian manifold and $X$ be a vector field on $M$. We consider the flow

$$
\frac{d x}{d t}=X(x)
$$

In order to change this dynamical system into a geodezic motion under a gyroscopic field of forces on a double dimension space, we build the quadratic Hamiltonian [11-12]

$$
\mathrm{H}(x, y)=\frac{1}{2}\|y\|^{2}-f(x)
$$

where $\|y\|^{2}=g(y, y)$ represents the Riemannian kinetic energy and

$$
f(x)=\frac{1}{2} g(X(x), X(x))=\frac{1}{2}\|X(x)\|^{2}
$$

is the Riemannian energy density of the vector field $X$.

The Hamiltonian $\mathcal{H}$ is conserved along the trajectories of the dynamics induced by the flow $d x / d t=X(x)$ in the sense of the following two rules:

1) if $\nabla$ is Levy-Civita connection of the Riemannian manidold $(M, g)$ with the components $G_{j k}^{i}$, then we differentiate the first order differential equation $d x / d t=X(x)$ with respect to $t$ and obtain

$$
\frac{\delta}{d t} \frac{d x^{i}}{d t}=\frac{d^{2} x^{i}}{d t^{2}}+G_{j k}^{i} \frac{d x^{j}}{d t} \frac{d x^{k}}{d t}
$$

2) on the other hand, the right hand member becomes

$$
\frac{\delta}{d t} X^{i}=\left(\nabla_{j} X^{i}\right) \frac{d x^{i}}{d t}=F_{j}^{i} \frac{d x^{j}}{d t}+\nabla^{i} f
$$

where $F_{j}^{i}=\nabla_{j} X^{i}-g^{i h} g_{k j} \nabla_{h} X^{k}$ is the external distinguished tensor field that characterizes the helicity of the vector field $X$, and $\nabla^{i} f=g^{i h} g_{k j}\left(\nabla_{h} X^{k}\right) X^{j}$ are the contravariant components of the conservative force $\nabla f$.

We obtain a single-time geometric dynamics [11-12]

$$
\frac{d^{2} x^{i}}{d t^{2}}+G_{j k}^{i} \frac{d x^{j}}{d t} \frac{d x^{k}}{d t}=F_{j}^{i} \frac{d x^{j}}{d t}+\nabla^{i} f
$$

The gyroscopic force $F_{j}^{i} d x^{j} / d t+\nabla^{i} f$ consists in the gyroscopic term $F_{j}^{i} d x^{j} / d t$ and the conservative term $\nabla^{i} f$.

Theorem 2. Every solution of second order ODEs system is a horizontal geodesic of the Riemannian-Lagrangian manifold

$$
\left(R \times M, 1+g, N_{1 j}^{i}=G_{j k}^{i} y^{k}-F_{j}^{i}\right)
$$

\section{Stokes Representation for the Solutions of Modified Maxwell-Vlasov PDEs System}

We consider next that electric field $E$ and magnetic field $B$ do not depend explicitly on the variable time $t$. Replacing the classical gyroscopic force $E+1 / c v \times B$ from Maxwell-Vlasov PDE's (1) with the gyroscopic force $F_{j}^{i} v^{j}+\nabla^{i} f$ from geometric dynamics, for the vectorial field $X=E$ and Riemannian metric $g_{i j}=\delta_{i j}$, and because curl E) $=0$, we obtain the modified MaxwellVlasov PDEs

$$
\begin{aligned}
& v_{1} \frac{\partial L}{\partial x_{1}}+v_{2} \frac{\partial L}{\partial x_{2}}+v_{3} \frac{\partial L}{\partial x_{3}} \\
& +\frac{e}{m}\left(\frac{\partial f}{\partial x_{1}} \frac{\partial f}{\partial v_{1}}+\frac{\partial f}{\partial x_{2}} \frac{\partial f}{\partial v_{2}}+\frac{\partial f}{\partial x_{3}} \frac{\partial f}{\partial v_{3}}\right)=0
\end{aligned}
$$

where $f$ is density of electric energy, $f=1 / 2\|E\|^{2}$.

Theorem 3. Let $\Omega_{\left(x_{0}, v_{0}\right),\left(x_{f}, v_{f}\right)}$ be the parallelepiped defined by diagonal points $\left(x_{0}, v_{0}\right)$ and $\left(x_{f}, v_{f}\right)$ and $n=(\tilde{N}, \bar{N})$ be the normal unit vector of the boundary $\partial \Omega_{\left(x_{0}, v_{0}\right),\left(x_{f}, v_{f}\right)}$. If $p(x)=\left(p_{i}(x)\right)_{i}, \quad q(x)=\left(q_{i}(x)\right)_{i}$, $\alpha(x), \beta(x), \gamma(x)$ are Stokes potentials, with $\operatorname{grad}_{v} \gamma=0$ and $\left.\gamma\right|_{\partial \Omega_{\left(x_{0}, v_{0}\right),\left(x_{f}, v_{f}\right)}}=0$, then the solutions of modified Maxwell-Vlasov PDEs (7) admit Stokes representation

$$
\begin{gathered}
E=\operatorname{curl}(p(x))-\operatorname{grad}(\alpha(x)) \\
B=\operatorname{curl}(q(x))-\operatorname{grad}(\beta(x))
\end{gathered}
$$

with condition $\left\langle\operatorname{grad}_{v} \gamma, v\right\rangle=0$, and boundary conditions

$$
\begin{aligned}
& \alpha \tilde{N}+p \times \tilde{N}+\left.\frac{e}{m} \gamma L \frac{\partial E}{\partial x} \bar{N}\right|_{\partial \Omega_{\left(x_{0}, v_{0}\right),\left(x_{f}, v_{f}\right)}}=0, \\
& \beta \tilde{N}+q \times\left.\tilde{N}\right|_{\partial \Omega_{\left(x_{0}, v_{0}\right),\left(x_{f}, v_{f}\right)}=0,}=0 \\
& \gamma\langle v, \tilde{N}\rangle+\left.\frac{e}{m} \frac{\partial f}{\partial x} \bar{N}\right|_{\partial \Omega_{\left(x_{0}, v_{0}\right),\left(x_{f}, v_{f}\right)}=0 .}=0
\end{aligned}
$$

Proof. We consider the following optimal control problem

$$
\max _{E(\cdot)} I(E(\cdot))=-\frac{1}{2} \int_{\Omega_{\left(x_{0}, v_{0}\right),\left(x_{f}, v_{f}\right)}}\left(\|E(x)\|^{2}+\|B(x)\|^{2}+\|\nu\|^{2}\right) d x d v
$$


subject to modified Maxwell-Vlasov PDE's (7), where $E(x)=\left(E_{i}(x)\right)_{i=1,3}$ is the electric $\mathrm{C}^{1}$ control vector function, $B(x)=\left(B_{i}(x)\right)_{i=\overline{1,3}}$ and $v=\left(v_{i}\right)_{i=1, \overline{3}}$ are $\mathrm{C}^{1}$ state vector functions, $B\left(x_{0}\right)=B_{0}, B\left(x_{f}\right)=B_{f}$, $v\left(x_{0}\right)=v_{0}, v\left(x_{f}\right)=v_{f}$.

Analogous with the proof of Theorem 1, necessary optimal conditions are equivalent with

$$
\begin{aligned}
& -E+\operatorname{curl} p-\operatorname{grad} \alpha-\frac{e}{m} L \operatorname{grad}_{v} \gamma \frac{\partial E}{\partial x}=0, \\
& -B+\operatorname{curl} p-\operatorname{grad} \beta=0, \\
& \left\langle\operatorname{grad}_{x} \gamma, v\right\rangle-\frac{e}{m}\left\langle\operatorname{grad}_{v} \gamma, \operatorname{grad} f\right\rangle=0, \\
& E_{1} \frac{\partial \gamma}{\partial v_{1}}=0, E_{2} \frac{\partial \gamma}{\partial v_{2}}=0, E_{3} \frac{\partial \gamma}{\partial v_{3}}=0, \\
& E_{2} \frac{\partial \gamma}{\partial v_{1}}+E_{1} \frac{\partial \gamma}{\partial v_{2}}=0, E_{3} \frac{\partial \gamma}{\partial v_{2}}+E_{2} \frac{\partial \gamma}{\partial v_{3}}=0, \\
& E_{1} \frac{\partial \gamma}{\partial v_{3}}+E_{3} \frac{\partial \gamma}{\partial v_{1}}=0
\end{aligned}
$$

satisfying the boundary conditions

$$
\begin{aligned}
& \alpha \tilde{N}+p \times \tilde{N}+\left.\frac{e}{m} \gamma L \frac{\partial E}{\partial x} \bar{N}\right|_{\partial \Omega_{\left(x_{0}, v_{0}\right),\left(x_{f}, v_{f}\right)}}=0, \\
& \beta \tilde{N}+q \times\left.\tilde{N}\right|_{\partial \Omega_{\left(x_{0}, v_{0}\right),\left(x_{f}, v_{f}\right)}}=0, \\
& \gamma\langle v, \tilde{N}\rangle+\left.\frac{e}{m} \frac{\partial f}{\partial x} \bar{N}\right|_{\partial \Omega_{\left(x_{0}, v_{0}\right),\left(x_{f}, v_{f}\right)}}=0, \\
& \left.E_{1} \gamma L n^{4}\right|_{\partial \Omega_{\left(x_{0}, v_{0}\right),\left(x_{f}, v_{f}\right)}}=0, \\
& \left.E_{2} \gamma L n^{5}\right|_{\partial \Omega_{\left(x_{0}, v_{0}\right),\left(x_{f}, v_{f}\right)}}=0, \\
& \left.E_{3} \gamma \operatorname{Ln}^{6}\right|_{\partial \Omega_{\left(x_{0}, v_{0}\right),\left(x_{f}, v_{f}\right)}}=0, \\
& \left.\gamma L\left(E_{2} n^{4}+E_{1} n^{5}\right)\right|_{\partial \Omega_{\left(x_{0}, v_{0}\right),\left(x_{f}, v_{f}\right)}=0}=0 \\
& \left.\gamma L\left(E_{3} n^{5}+E_{2} n^{6}\right)\right|_{\partial \Omega_{\left(x_{0}, v_{0}\right),\left(x_{f}, v_{f}\right)}}=0, \\
& \left.\gamma L\left(E_{1} n^{6}+E_{3} n^{4}\right)\right|_{\partial \Omega_{\left(x_{0}, v_{0}\right),\left(x_{f}, v_{f}\right)}}=0
\end{aligned}
$$

where $n=(\tilde{N}, \bar{N})$ is the normal unit vector of the boundary $\partial \Omega_{\left(x_{0}, v_{0}\right),\left(x_{f}, v_{f}\right)}, \quad \bar{N}=\left(n^{i}\right)_{i=\overline{4,5}}, \quad \tilde{N}=\left(n^{j}\right)_{j=1, \overline{3}}$.

Since $\operatorname{grad}_{v} \gamma=0$ and $\left.\gamma\right|_{\partial \Omega_{\left(x_{0}, v_{0}\right),\left(x_{f}, v_{f}\right)}}=0$ from the hypotheses, it results Stokes representation (8), (9), for the solutions of Maxwell-Vlasov modified PDEs (7) together with the boundary conditions (10).

\section{Conclusions}

The present paper studies the electro-magnetic dynamic systems from the perspective of optimal control theory (see also $[5,11,18]$ ). Multi-time optimal control problems when the functional is represented by the density of the electromagnetic energy, subject to Maxwell PDE's or modified Maxwell-Vlasov PDE's (classical gyroscopic force $E+1 / c v \times B$ from Maxwell-Vlasov PDE's is replaced with the gyroscopic force $F_{j}^{i} v^{j}+\nabla^{i} f$ from geometric dynamics, for the vectorial field $X=E$ and Riemannian metric $g_{i j}=\delta_{i j}$ ) reliefy electromagnetic evolutions from Stokes representations. The study of electromagnetic energy in magnetic traps justify our research.

\section{Acknowledgements}

We have benefited greatly from conversations with Prof. Dr. V. Prepeliță and Prof. Dr. I. Țevy.

\section{REFERENCES}

[1] C. Udrişte, "Geometric Dynamics," Kluwer Academic Publishers, Article Southeast Asian Bulletin of Mathematics, Springer-Verlag, New York, Vol. 24, 2000, pp. 313-322.

[2] C. Udrişte, "Nonclassical Lagrangian Dynamics and Potential Maps," Proceedings of the Conference on Mathematics in Honour of Profesor Radu Roşca at the Ocassion of His Ninetieth Birthday, Belgium, 11-16 December 1999.

[3] C. Udrişte and M. Postolache, "Atlas of Magnetic Geometric Dynamics," Geometry Balkan Press, Bucharest, 2001.

[4] C. Udrişte, "Tools of Geometric Dynamics," Buletinul Institutului de Geodinamică, Academia Româna, Vol. 14, No. 4, 2003, pp. 1-26.

[5] C. Udrişte, "Maxwell Geometric Dynamics," European Computing Conference, Athens, Greece, 24-26 September 2007, pp. 1597-1609.

[6] C. Udrişte and M. Pîrvan, "Magnetic Dynamics around a Configuration of Two Square AntiHelmholtz Coils," Proceedings of 7th WSEAS International Conference Nonlinear Analysis, Non-linear Systems and Chaos (NOLASC 08), Corfu, Greece, 26-28 October 2008, pp. 222-227.

[7] C. Udrişte and M. Pîrvan, "Magnetic Geometric Dynamics around Tornado Trap," Proceedings of the 10th WSEAS International Co nference Mathematical Computational Mathematical Science and Engineering (MACMESE 08), Bucharest, 7-9 November 2008, pp. 312-317.

[8] P. Preuss, "Angels, Demons, and Antihydrogen: The Real Science of Anti-Atoms," 2009. http://www.symmetry magzine.org/breaking/2009/05/07/angels-demons-and-ant ihydrogen-the-rel-science-of-anti-atoms/

[9] C. Udrişte and L. Matei, "Teorii Lagrange-Hamilton," (in 
Romanian) Monographs and Textbooks 8, Geometry Balkan Press, Bucharest, 2008.

[10] C. Udrişte, "Simplified Multitime Maximum Principle," Balkan Journal of Geometry and Its Applications, Vol. 14, No. 1, 2009, pp. 102-119.

[11] C. Udrişte, M. Ferrara and D. Opriş, "Economic Geometric Dynamics," Monographs and Textbooks 6, Geometry Balkan Press, Bucharest, 2004.

[12] C. Udrişte, "Geodesic Motion in a Gyroscopic Field of Forces,” Tensor, N. S., Vol. 66, No. 3, 2005, pp. 215-228.

[13] H. Cendra, D. D. Holm, M. J. W. Hoyle and J. E. Marsden, "The Maxwell-Vlasov Equations in Euler-Poincaré form," Journal of Mathematical Physics, Vol. 39, No. 6, 1998, pp. 3138-3157. doi:10.1063/1.532244

[14] V. Ciancio and C. Udrişte, "Ioffe-Ştefănescu Magnetic Trap," Revue Roumaine de Sciences Techniques-Serie Electrotechnique et Energetique, Vol. 49, No. 2, 2004, pp. 157-176.

[15] Y. Guo, "Global Weak Solutions of the Vlasov-Maxwell
System with Boundary Conditions," Communications in Mathematical Physics, Vol. 154, No. 2, 1993, pp. 245263. doi:10.1007/BF02096997

[16] E. Marsden and T. S. Raţiu, "Intro-Duction to Mechanics and Symmetry," 2nd Edition, Springer, New York, 1999.

[17] C. Neagu and C. Udrişte, "From PDE Systems and Metrics to Geometric Multi-time Field Theories," Seminarul de Mecanică, Sisteme Dinamice Diferențiale, 79, Universitatea de Vest din Timişoara, 2001.

[18] M. Pîrvan and C. Udrişte, "Optimal Control of Electromagnetic Energy," Balkan Journal of Geometry and Its Applications, Vol. 15, No. 1, 2010, pp. 131-141.

[19] C. Udrişte, "Nonholonomic Aapproach of Multitime Maximum Principle," Balkan Journal of Geometry and Its Applications, Vol. 14, No. 2, 2009, pp. 101-116.

[20] D. Wang and X. Hu, "Weak Stability and Hydrodynamic Limit of Vlasov-Maxwell-Bolzmann Equations," SIAM Conference on Analysis of Partial Differential Equations, Miami, Florida, 7-10 December 2009, p. 78. 